\title{
Illumination effects in holographic imaging of the electrostatic potential of defects and $p n$ junctions in transmission electron microscopy
}

\author{
L. Houben, ${ }^{1}$ M. Luysberg, ${ }^{1}$ and T. Brammer ${ }^{2}$ \\ ${ }^{1}$ Institut für Festkörperforschung, Forschungszentrum Jülich, 52425 Jülich, Germany \\ ${ }^{2}$ Institut für Photovoltaik, Forschungszentrum Jülich, 52425 Jülich, Germany
}

(Received 5 January 2004; published 18 October 2004)

\begin{abstract}
Off-axis holography has successfully revealed the built-in potential in highly doped $p n$-junctions, making holographic phase retrieval in transmission electron microscopy (TEM) a prospective tool for imaging the electrostatic potential in a semiconductor sample or device. The effect of electron-hole pair generation during electron illumination and the presence of defect rich amorphized sample surfaces in a TEM sample on the electrostatic potential is investigated for the test case of an electrically active grain boundary and a $p n$-junction in silicon by numerical simulation. In the case of the grain boundary, the pair generation in the electron beam leads to significantly increased recombination currents into trap state defects. As a result, the trapped charge in the defects is decreased and the potential drop around the electrically active defect is considerably reduced, for electron-hole pair generation rates typically present in a TEM experiment even below the detection limit of holography. In the case of $p n$-junctions distorted potential maps are predicted for dopant densities smaller than $10^{17} \mathrm{~cm}^{-3}$.
\end{abstract}

DOI: 10.1103/PhysRevB.70.165313

PACS number(s): 61.14.Nm

\section{INTRODUCTION}

Holographic imaging in transmission electron microscopy (TEM) has proven to be a powerful tool for imaging variations in a sample's inner potential $V_{0}$. Electron wave phase images taken by off-axis holography of $p n$-junctions in FET devices, ${ }^{1,2}$ and measurements of the phase maps of biased bipolar $p n$-junctions $s^{3}$ have demonstrated that the method is capable of reproducively revealing small spatial variations in the electrostatic potential of dopant profiles.

Accuracy limits for the method are described mostly in terms of instrumentation limits and imaging artifacts specific to the method as well as detection limits, especially for the case of high resolution off-axis holography. ${ }^{4,5}$ Artifacts related to sample imperfectness are also described in literature: surface charging and surface "dead layers" $", 3$ had to be considered for a proper quantification of the $p n$-junction potential. Both factors are reported to be sensitive to specimen preparation. ${ }^{1,3,6}$ It will be shown in this paper that the quantification can be further complicated by the the invasive nature of the experiment, namely the interaction of the irradiating electron beam with the sample. Especially the interaction between electrically active defects at structurally or chemically heterogeneous interfaces and electrongenerated excess charge carriers has to be taken into account.

An example where the phase map translated into an electrostatic potential map contradicts expectations is given in Fig. 1. The $1 \mathrm{k} \times 1 \mathrm{k}$ CCD image in Fig. 1(a) shows an offaxis hologram taken from a nearly symmetrical $\langle 110\rangle$ tilt boundary with a small twist component of approx. $2^{\circ}$ in a Czochralski-grown silicon bicrystal. Figures 1(b) and 1(c) shows the amplitude and the phase of the transmitted electron wave, corrected by the vacuum reference hologram (see, e.g., Ref. 4 for a description of the reconstruction procedure). The amplitude profiles perpendicular to the grain boundary (Fig. 2) show little amplitude variation since care was taken to assure kinematical diffraction conditions. The peak phase shift of 1.1 radians (Fig. 2) in the phase image can be translated into a potential drop of the the inner potential of approximately $1.2 \mathrm{eV}$, using the phase change from the sample edge to the vacuum and the experimentally determined mean inner potential of $9.3 \mathrm{eV}$ for bulk silicon ${ }^{7}$ for calibration. The phase map, therefore, implies the result of a potential drop larger than the band-gap energy. Furthermore, the width of the phase change peak around the grain boundary is significantly smaller than the typical screening length for the potential of charged particles (Debye-length) of approximately $150 \mathrm{~nm}$ for the doping level in the order of $10^{16} \mathrm{~cm}^{-3}$. The mismatch in the potential barrier height and width, therefore, make the assignment of the phase image to a grain boundary potential barrier implausible.

Apart from structural inhomogeneities, which give rise to phase shifts of the electron wave due to compositional charge density modulations, the potential maps may be affected by a number of effects that render a TEM holography experiment an invasive type of experiment. First, the sample is a thin foil containing the previously mentioned surface defects which can be charged during the experiment. Second, the electron beam produces electron-hole pairs on its passage through the sample. Consequently, the spatial charge density associated with free charge carriers, defects and charged dopant sites may take a stationary equilibrium different from the undisturbed device. The experimentally acquired phase maps under illumination with high energy electrons will, therefore, not be linked directly to the dopant concentrations or defect charges in the undisturbed case.

For a concise quantification of the phase maps in terms of the electrostatic potential, the stationary charge density comprised of free charge carriers and spatially located charged defects and the electrostatic potential have to be calculated in a self-consistent manner including for charge carrier generation and recombination. The following theoretical study 
(a)

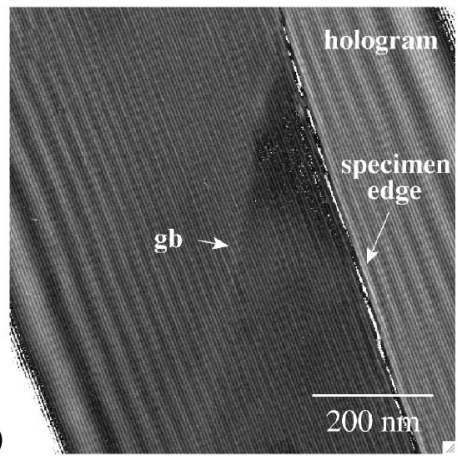

(b)

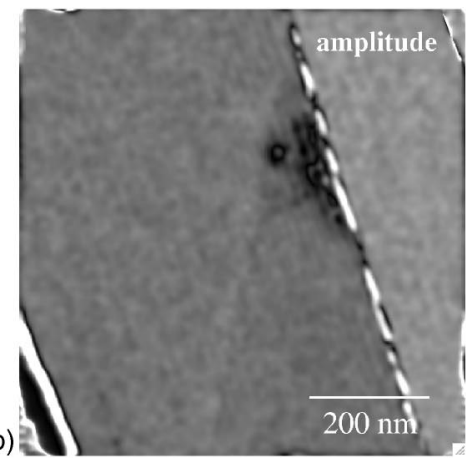

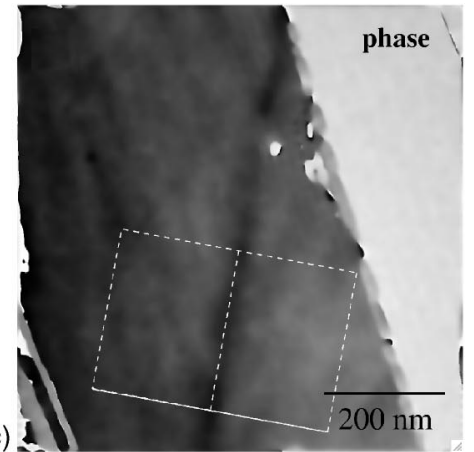

FIG. 1. (a) Off-axis hologram of a bicrystal grain boundary (gb) in silicon. (b) Amplitude and (c) phase reconstructed from the sideband in the FFT of the hologram.

demonstrates the effect of the charge carrier equilibration on the electrostatic potential around grain boundary defects and bipolar $p n$-junctions in silicon including both the surface effects and the illumination effect.

\section{NUMERICAL SIMULATION}

\section{A. Description of experiment}

Figure 3 gives a pictorial representation of the model which formed the basis for the simulation of the electrostatic potential and the charge carrier equilibration. The figure also depicts the relevant geometrical dimensions: The thicknesses $t$ of the sample and $t^{\prime}$ of the damaged surface layers, the distance $L / 2$ of the examined region from contacts to the specimen holder and the locally confined illumination spot of diameter $u$ where excess charge carriers are produced.

The following simplifications were made in the numerical simulations: (1) The surface of the silicon TEM sample is idealized as a heterojunction between crystalline silicon (c$\mathrm{Si}$ ) and amorphous silicon (a-Si). Single crystalline silicon parameters were attributed to the crystalline material and parameters typical for defect-rich amorphous silicon of poor electrical quality were assumed for the amorphous layer part.

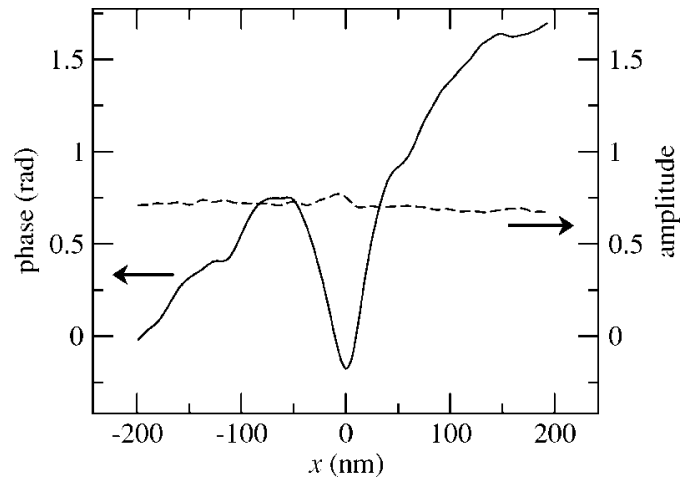

FIG. 2. Phase and amplitude profile taken perpendicular to the grain boundary in the box indicated in the phase image in Fig. 1(c). The amplitude of the hologram fringes is normalized to the amplitude in a reference vacuum hologram. The steep gradient in the phase profile is due to the thickness change in the wedge shaped TEM sample.
Representative data can be taken, e.g., from Ref. 8 for crystalline silicon and Ref. 9 for amorphous silicon. (2) The contacts at the sample boundary to the left and right show ohmic behavior, i.e., the charge carrier concentrations were kept constant at these contacts irrespective of the charge carrier generation.

The generation rate for electron-hole pair creation is assumed constant because of the high kinetic energy of primary electrons and the small sample thickness. The following section gives an estimation for the order of magnitude of the electron-hole pair generation rate in the sample.

\section{B. Generation rate for excess charge carriers under electron irradiation}

During its passage through the TEM sample, the high energy electron beam suffers from inelastic scattering. Apart from radiation damage in the form of displaced atoms and vacancies, the high-energy electron will lose energy due to ionization and leave a trail of electron-hole pairs in a semiconductor. In order to estimate the magnitude of the charge carrier generation terms for electrons $\left(G_{n}\right)$ and holes $\left(G_{p}\right)$, the energy loss as a function of the penetration depth $x$ can be derived from the Bethe-Bloch equation ${ }^{10}$

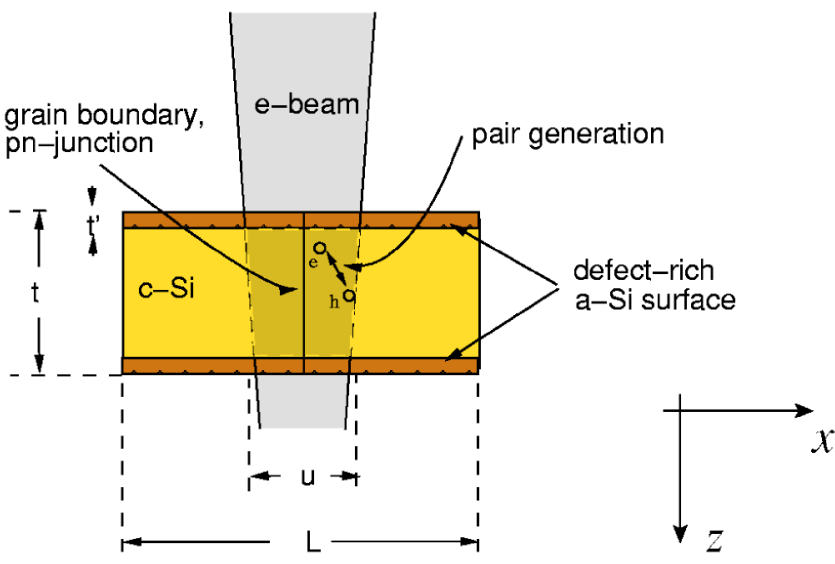

FIG. 3. Schematic model used for the numerical simulations. A thin TEM sample with a thickness $t$ and defect surface layers with thickness $t^{\prime}$ contains a singularity, e.g., a $p n$-junction or a grain boundary, in the center of the region of interest. Electron-hole pairs are generated in the region transmitted by the electron beam. 


$$
-\frac{\mathrm{d} E}{\mathrm{~d} x}=K x^{2} \frac{Z}{A} \frac{1}{\beta^{2}}\left(\frac{1}{2} \ln \frac{2 m_{e} c^{2} \beta^{2} \gamma^{2} E}{I^{2}}-\beta^{2}-\frac{\delta}{2}\right),
$$

where $\beta=v / c$ and $\gamma=E / m_{0} c^{2}$ and $\delta / 2$ is the mass density correction for high particle energies. According to Eq. (1) the average energy loss $\Delta E$ of a $200 \mathrm{kV}$ electron on its passage through a $100 \mathrm{~nm}$ thick silicon film amounts to $78.6 \mathrm{eV}$. This energy loss is mainly caused by plasmon creation and electron-hole pair creation. In this study, equipartition into plasmon energy loss and pair creation energy loss was assumed. Taking the typical electron-hole pair creation energy $I$ of $3.6 \mathrm{eV}$ one incident $200 \mathrm{kV}$ electron will then produce on average one electron hole pair after each $10 \mathrm{~nm}$ of penetration depth.

With an electron beam current density $j_{\mathrm{BC}}$ and a sample thickness $t$ the electron hole pair generation rate amounts to

$$
G_{n}=G_{p}=\frac{1}{2} \frac{{ }_{\mathrm{BC}}\left|\frac{\mathrm{d} E}{\mathrm{~d} x}\right|}{e I} .
$$

The beam current density of the electron probe in a Schottky type field-emission transmission electron microscope on the sample is typically in the order of a few nA confined to an area of a few $\mu \mathrm{m}^{2}$. Taking a sample thickness of $100 \mathrm{~nm}$, a beam current of $1 \mathrm{nA}$ and a circular illuminated area diameter of $2 \mu \mathrm{m}$ results in a generation rate $G=G_{n, p}$ $=8.6 \times 10^{23} \mathrm{~cm}^{-3} \mathrm{~s}^{-1}$.

In comparison, the generation rate under complete absorbance of an Air Mass 1.5 solar standard radiation spectrum in silicon corresponds to $G=2.6 \times 10^{21} \mathrm{~cm}^{-3} \mathrm{~s}^{-1}$. Thus, the electron-hole pair generation rate in a TEM is typically orders of magnitude higher than in an experiment with optical white light irradiation.

\section{Basic equations}

This section briefly surveys the basic equations for the numerical simulations. An indepth description is given in, e.g., the textbook by Selberherr (Ref. 8) on the numerical analysis of semiconductor devices. The electrostatic potential $\phi(\vec{r})$ and the charge densities of free electrons $n(\vec{r})$ and holes $p(\vec{r})$ were calculated by numerical solution of the Poisson equation coupled to the continuity equations for electrons and holes:

$$
\begin{gathered}
\Delta \phi(\vec{r})=\frac{e}{\varepsilon \varepsilon_{0}}\left(N_{\mathrm{D}}^{+}(\vec{r})-N_{\mathrm{A}}^{-}(\vec{r})+p(\vec{r})-n(\vec{r})+Q(\vec{r})\right), \\
\frac{\partial n(\vec{r})}{\partial t}=-\frac{1}{e} \nabla j_{n}(\vec{r})+G_{n}(\vec{r})-R_{n}(\vec{r}), \\
\frac{\partial p(\vec{r})}{\partial t}=\frac{1}{e} \nabla j_{p}(\vec{r})+G_{p}(\vec{r})-R_{p}(\vec{r}) .
\end{gathered}
$$

Here, $e$ is the elementary charge, $\varepsilon$ the vacuum dielectric permittivity and $\varepsilon_{0}$ the static dielectric permittivity. $N_{\mathrm{D}}^{+}(\vec{r})$ is the density of ionized donor atoms, and $N_{\mathrm{A}}^{-}(\vec{r})$ the density of ionized acceptor atoms. $Q(\vec{r})$ is an additional term represent- ing the charge trapped in defects. $G_{n}(\vec{r})$ and $G_{p}(\vec{r})$ are the volume generation rates for electrons and holes, respectively, and $R_{n}(\vec{r})$ and $R_{p}(\vec{r})$ their volume or interface recombination rates.

Carrier concentrations were calculated using Boltzmann statistics for nondegenerate semiconductors with parabolic band edges:

$$
n(\vec{r})=N_{C} \exp \left(-\frac{\mathcal{E}_{C}(\vec{r})+e \phi(\vec{r})-\eta(\vec{r})}{k_{B} T}\right),
$$

with the Boltzmann constant $k_{B}$ and the equilibrium temperature $T . N_{C}$ is the effective density of states at the conduction band edge and $\mathcal{E}_{C}(\vec{r})$ the conduction band edge energy and $\eta$ the quasi-Fermi energy of the electrons.

Similarly, with the effective density of states $P_{V}$ at the valence band edge, the valence band energy $\mathcal{E}_{V}(\vec{r})$ and the hole quasi-Fermi energy $\psi$ :

$$
p(\vec{r})=P_{V} \exp \left(\frac{\mathcal{E}_{V}(\vec{r})+e \phi(\vec{r})-\psi(\vec{r})}{k_{B} T}\right) .
$$

The drift-diffusion current densities for electrons, $j_{n}(\vec{r})$, and for holes, $j_{p}(\vec{r})$, are given within the framework of the Boltzmann transport theory by the classical approximation

$$
\begin{gathered}
j_{n}(\vec{r})=-e n \mu_{n} \nabla \phi(\vec{r})-e D_{n} \nabla n(\vec{r}), \\
j_{p}(\vec{r})=e p \mu_{p} \nabla \phi(\vec{r})-e D_{p} \nabla p(\vec{r}) .
\end{gathered}
$$

Here, $\mu_{n}$ and $\mu_{p}$ are the electron and hole effective mobility, $D_{n}=-\mu_{n}\left(k_{B} T / e\right)$ and $D_{p}=\mu_{p}\left(k_{B} T / e\right)$ their diffusion constants.

Trap assisted extrinsic recombination dominates in indirect band-gap semiconductors and the recombination rates $R_{n(p)}$ where therefore calculated using the Schockley-ReadHall (SRH) recombination rate equations ${ }^{11,12}$ for mid-gap defects. Ohmic contacts and surface recombination terms were introduced to satisfy the boundary conditions at the model boundary or inner interfaces. The surface recombination rates were calculated from a modified SRH recombination expression for trap assisted recombination, parametrized by surface recombination velocities $v_{\mathrm{e}}$ for electrons and $v_{\mathrm{h}}$ for holes. ${ }^{13}$ For numeric computation, the set of Eqs. (3)-(5) was solved iteratively using a finite difference approach following the Scharfetter and Gummel approach (see Refs. 8 and 14 for a detailed description) with nonequidistant mesh spacing.

\section{SIMULATION RESULTS}

The numerical simulations were separated into two onedimensional problems in order to reduce the complexity. The first one deals with the effect of a surface damage layers on a homogeneous crystalline silicon layer. The spatial coordinate for this model is parallel to the transmission direction, i.e., the $z$-coordinate in Fig. 3. The second set of simulations treats the influence of the electron-hole pair generation on potential maps of a defect structure or a dopant step profile orthogonal to the transmission direction. 
TABLE I. Material parameters used in the one dimensional simulation of the three-layer a-Si/c-Si/a-Si stack. $t$ is the layer thickness, $\mathcal{E}_{\text {gap }}$ the band-gap energy, $N_{\mathrm{A}}$ the acceptor dopant density, $D_{0}$ the density of defect states, $\tau_{\mathrm{n}}$ and $\tau_{\mathrm{p}}$ the electron and hole lifetime and $\mu_{\mathrm{n}}$ and $\mu_{\mathrm{p}}$ their effective mobility (Ref. 15).

\begin{tabular}{ccccccccc}
\hline \hline & $\frac{t}{\mathrm{~nm}}$ & $\frac{\mathcal{E}_{\text {gap }}}{\mathrm{eV}}$ & $\frac{N_{\mathrm{A}}}{\mathrm{cm}^{-3}}$ & $\frac{D_{0}}{\mathrm{~cm}^{-3}}$ & $\frac{\tau_{\mathrm{n}}}{\mu \mathrm{s}}$ & $\frac{\tau_{\mathrm{p}}}{\mu \mathrm{s}}$ & $\frac{\mu_{\mathrm{n}}}{\mathrm{V} \mathrm{cm}^{2} \mathrm{~s}^{-1}}$ & $\frac{\mu_{\mathrm{p}}}{\mathrm{V} \mathrm{cm}^{2} \mathrm{~s}^{-1}}$ \\
\hline $\mathrm{c}-\mathrm{Si}$ & 150 & 1.1 & $10^{16}, 10^{19}$ & $10^{15}$ & 10 & 10 & 1400 & 400 \\
$\mathrm{a}-\mathrm{Si}$ & 3 & $1.7,1.1$ & $10^{16}, 10^{19}$ & $10^{19}$ & $10^{-5}$ & $10^{-5}$ & 10 & 4 \\
\hline \hline
\end{tabular}

\section{A. The effect of amorphous surface layers on the density of excess charge carriers}

For the simulation of surface effects, a model of three stacked layers consisting of $3 \mathrm{~nm}$ amorphous silicon, $150 \mathrm{~nm}$ crystalline silicon and another $3 \mathrm{~nm}$ of amorphous silicon was chosen. The thickness of the crystalline layer was chosen to be in the range providing a good signal-to-noise ratio for the phase images related to the voltage drop at $p n$-junctions in silicon. The thickness of $3 \mathrm{~nm}$ for the surface amorphous layer can be seen as a typical value for the depth of the amorphization in the surface layers of a TEM sample due to the ion beam damage introduced during argon ion milling. ${ }^{16}$ In all cases simulated, a depth independent pair generation rate $G=1 \times 10^{21} \mathrm{~cm}^{-3} \mathrm{~s}^{-1}$ was applied. The surface recombination velocity in the a-Si surface is assumed to be equal for electrons and holes and takes the value of the thermal velocity $s=1 \times 10^{7} \mathrm{~cm} \mathrm{~s}^{-1}$. Quasi-neutrality is maintained. Further material parameters are presented in Table I.

Figure 4 displays the spatial density of free electrons $n(x)$ and free holes $p(x)$ for four different cases with $\langle p\rangle$-type material with dopant densities $N_{\mathrm{A}}$ equal to $10^{19}$ and $10^{16} \mathrm{~cm}^{-3}$ and different band-gap energies in the amorphous silicon and different band edge alignment. The choice of different band-offsets for the simulation is motivated by the controversial data given in literature (see, e.g., discussion in Ref. 17). Here, a difference $\Delta$ of $0.6 \mathrm{eV}$ in the fundamental energy gap in crystalline silicon and device-grade amorphous silicon was assumed. $\Delta$ was split into a valence band discontinuity of $\frac{1}{3} \Delta$ and a conduction band offset of $\frac{2}{3} \Delta$. In another set of simulation parameters, the energy gap of the indirect gap of crystalline silicon was ascribed to the amorphous layers in order to simulate the special case without band gap
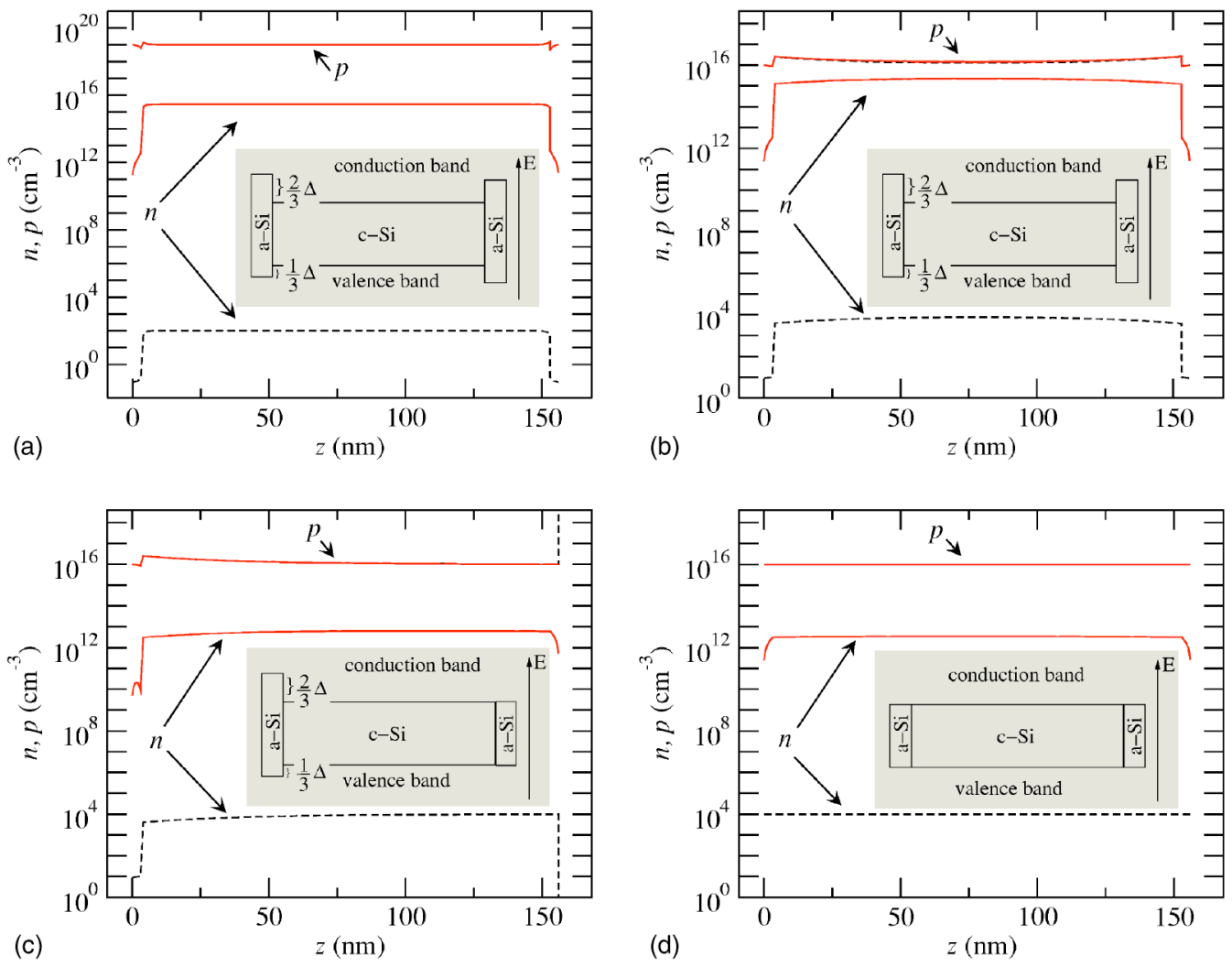

FIG. 4. Stationary charge carrier density $n$ of free electrons and $p$ of free holes in a $150 \mathrm{~nm}$ thin layer of crystalline silicon covered by $3 \mathrm{~nm}$ thick amorphous layers in the nonirradiated (dashed line) and in the irradiated case (solid line). The insets show details of the band-gap alignment for the a-Si/c-Si heterojunctions, where $\Delta=0.6 \mathrm{eV}$ is the difference in the fundamental gap between crystalline and device grade amorphous silicon. (a) $N_{A}=1 \times 10^{19} \mathrm{~cm}^{-3}$ (b)-(d) $N_{A}=1 \times 10^{16} \mathrm{~cm}^{-3}$. 
TABLE II. Parameters used in the one dimensional simulation of a grain boundary. $\frac{1}{2} L$ is the distance between the grain boundary and the ohmic contacts at the sides of the sample, $u$ is the diameter of the electron illumination spot, $N_{\mathrm{A}}$ the acceptor dopant density. $D$ represents the density of interface defect states, $E_{\text {def }}$ is the mid position of the Gaussian defect profile above the valence band edge with a standard deviation $\sigma_{\text {def }} \cdot v_{\mathrm{e}}$ and $v_{\mathrm{h}}$ are the interface recombination velocities for electrons and holes.

\begin{tabular}{cccccccc}
\hline \hline$\frac{L}{\mu \mathrm{m}}$ & $\frac{u}{\mu \mathrm{m}}$ & $\frac{N_{\mathrm{A}}}{\mathrm{cm}^{-3}}$ & $\frac{D}{\mathrm{~cm}^{-2}}$ & $\frac{E_{\mathrm{def}}}{\mathrm{eV}}$ & $\frac{\sigma_{\mathrm{def}}}{\mathrm{eV}}$ & $\frac{v_{\mathrm{e}}}{\mathrm{s}^{-1} \mathrm{~cm}}$ & $\frac{v_{\mathrm{h}}}{\mathrm{s}^{-1} \mathrm{~cm}}$ \\
\hline 10,100 & 2 & $10^{16}$ & $3 \times 10^{11}-1 \times 10^{12}$ & 0.55 & 0.1 & $10^{7}$ & $10^{7}$ \\
\hline \hline
\end{tabular}

offset between the crystalline and the amorphous layers.

The following observations can be made for the homogeneously illuminated sample: The concentration of the majority carriers is enhanced only insignificantly even at the lowest dopant density of $N_{\mathrm{A}}=10^{16} \mathrm{~cm}^{-3}$. Contrary, the minority carrier density raises by orders of magnitude. Especially in the case shown in Fig. 4(b), where a band gap discontinuity restrains the generated charge carriers from entering the amorphous surface layers and recombining there. The maximum value $n_{\max }$ of the minority charge carrier density reaches $2 \times 10^{15} \mathrm{~cm}^{-3}$ here, which is just one order of magnitude less than the density of the majority carriers.

When neglecting recombination and carrier transport, the product of generation rate and carrier lifetime, $G \tau$, can serve as a rough estimate for the excess charge carrier concentrations. $G \tau$ equals to $1 \times 10^{16} \mathrm{~cm}^{-3}$ in all cases shown. In the case of a band-gap discontinuity [Figs. 4(a) and 4(b)] at both surfaces, the peak value $n_{\max }$ of the excess minority charge carrier density is close to $G \tau$. If no bandgap discontinuity is assumed [Figs. 4(c) and 4(d)], $n_{\max }$ is only about two to three orders of magnitude lower than $G \tau$. Even in this latter case, $n_{\max }$ is not substantially reduced by the presence of the surface layers.

\section{B. The electrostatic potential at grain boundaries}

In order to investigate the impact of the excess charge carrier generation under the electron beam on the electrostatic potential at a grain boundary, a one dimensional model comprising a spatially delta-shaped defect peak at its center was chosen. The defect peak corresponds to a distribution of defect states within the band-gap associated with a grain boundary containing dangling bonds.

A Gaussian energy distribution of defect states around the mid-gap position and a standard deviation of $0.1 \mathrm{eV}$ was used to realize the prevalent description for a grain boundary containing intrinsic defects (see, e.g., Ref. 18, and references therein). The electrostatic charge $Q(\vec{r}=0)$ associated with the defect states was recalculated on the basis of the Fermi-Dirac statistics in each cycle of the iterative solution of Eqs. (3)-(5).

Ohmic contacts form the boundary of the model with length $L$, i.e., the distance between the defect and the contacts is $\frac{1}{2} L$. A Gaussian generation rate profile around the defect was assumed. The standard deviation of this generation profile was adapted to $u$ and the area normalized to the generation rate $G$. Calculations were done for various interfacial trap state densities $D$ at the grain boundary, contact distances $\frac{1}{2} L$ and generation rates $G$. The acceptor dopant density was fixed at $1 \times 10^{16} \mathrm{~cm}^{-3}$. Lifetime and mobility parameters for the crystalline silicon can be taken from Table I, additional parameters in this simulation are listed in Table II. Figure 5 displays the influence of the electron-hole pair generation on the stationary charge carrier density of electrons $n$ and holes $p$, their electro-chemical potential $\eta-e \phi$ and $\psi+e \phi$, respectively, and the electrostatic potential energy $e \phi$ at a grain boundary with a defect density $D=1$ $\times 10^{12} \mathrm{~cm}^{-2}$. Upon electron-hole pair generation, the concentration $n$ of the minority carriers is increased significantly [Fig. 5(a)]. A similar increase in the hole concentration can be seen on a linear scale in Fig. 5(b). The electro-chemical potential of holes and electrons splits in the illuminated spot with a diameter of $2 \mu \mathrm{m}$ around the grain boundary position whereas it is pinned at the ohmic contacts contacts [Fig. 5(c)]. Excess minority carriers are attracted by the grain boundary potential, leading to an enhanced recombination current and a re-charging of defects. As a consequence, the grain boundary potential barrier height $\phi_{\mathrm{B}}=\phi(0)$ is reduced.

The decrease of the barrier height $e \phi_{\mathrm{B}}$ with increasing generation rate $G$ is shown in Fig. 6. Generation rates $G$ greater than $1 \times 10^{22} \mathrm{~cm}^{-3} \mathrm{~s}^{-1}$, easily attained in a TEM, lead to an effective suppression of the potential barrier. The balanced barrier height $e \phi_{\mathrm{B}}$ is related to the maximum in the recombination currents for the SRH interface trap state recombination.

\section{Charge carrier distribution at $p n$-junctions}

A one-dimensional model with an abrupt change in dopant concentration in its center was used to analyze the charge carrier equilibrium concentrations and the electrostatic potential at a $p n$-junction. The dopant concentrations satisfy the following relations:

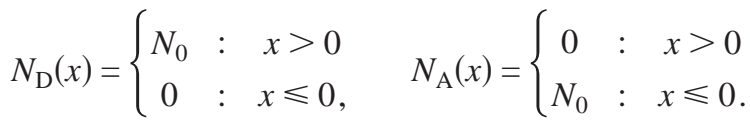

The energy gap, lifetime and mobility parameters for crystalline silicon can be taken from Table I.

Figure 7 shows exemplary the charge carrier density, electro-chemical potential and electro-static potential at a $p n$ junction with a doping level $N_{0}=1 \times 10^{16} \mathrm{~cm}^{-3}$ and various generation rates $G$. The distance between the junction position and the ohmic contacts is $50 \mu \mathrm{m}$ in this case. The increase of the excess charge carrier concentration with gen- 

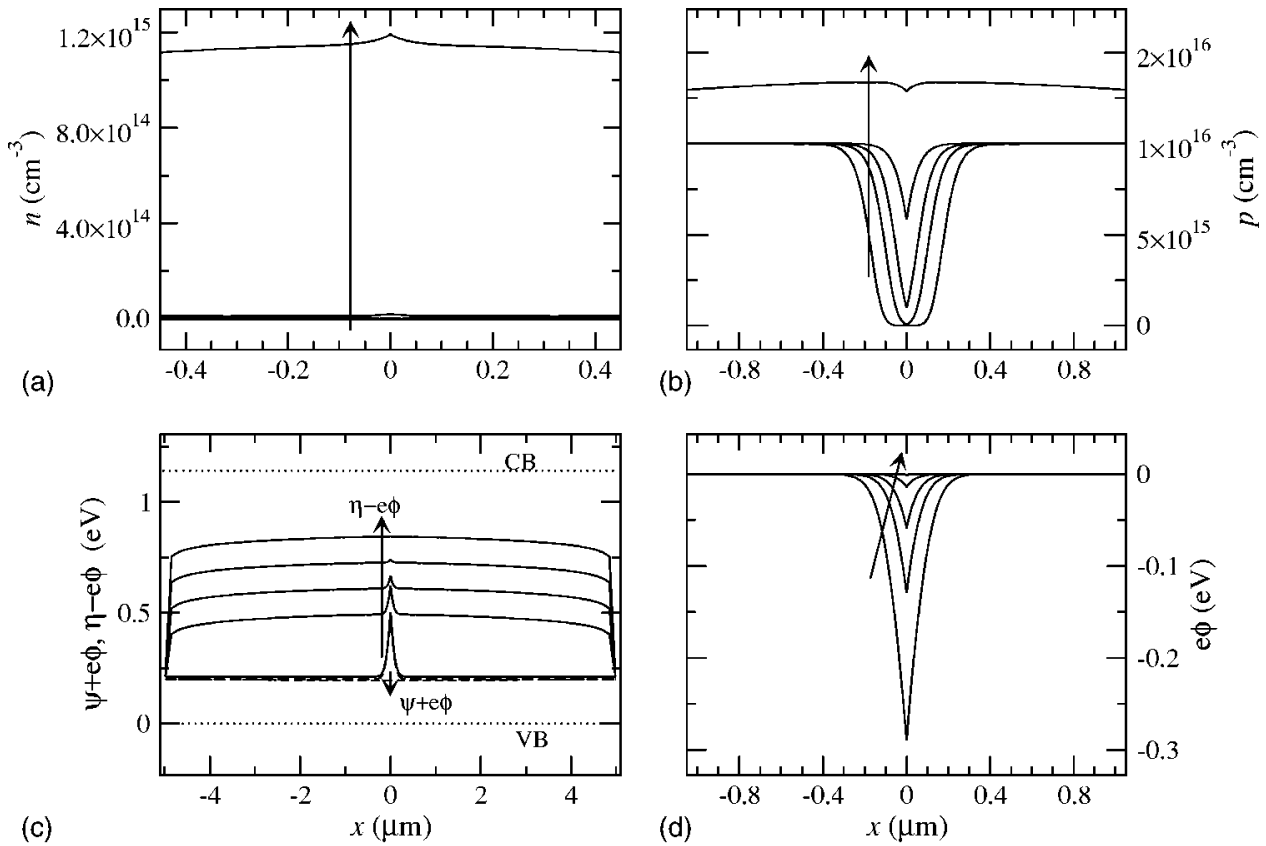

FIG. 5. The effect of excess charge carrier generation under electron beam irradiation on (a) the stationary charge carrier density $n$ of electrons and (b) $p$ of holes, (c) the electro-chemical potential $\eta-e \phi$ of electrons (solid lines) and $\psi+e \phi$ of holes (dashed lines) and (d) the electrostatic potential $e \phi$ at a grain boundary decorated with trap states. The electro-chemical potential $\psi+e \phi$ shows little change in the logarithmic plot in (c). Calculations were performed for an acceptor doping $N_{A}=1 \times 10^{16} \mathrm{~cm}^{-3}$ and boundary trap state density $D=1$ $\times 10^{12} \mathrm{~cm}^{-2}$. Curves are plotted for excess charge carrier generation rate $G=0 \mathrm{~cm}^{-3} \mathrm{~s}^{-1}, 1 \times 10^{18} \mathrm{~cm}^{-3} \mathrm{~s}^{-1}, 1 \times 10^{20} \mathrm{~cm}^{-3} \mathrm{~s}^{-1}, 1$ $\times 10^{22} \mathrm{~cm}^{-3} \mathrm{~s}^{-1}$ and $1 \times 10^{24} \mathrm{~cm}^{-3} \mathrm{~s}^{-1}$, with arrows indicating the change upon increasing electron-hole pair generation rate $G$.

eration rate $G$ is clearly observable in the electro-chemical potential plots in Fig. 7(c) and for the higher generation rates also from the linear plots of the charge carrier densities in (a) and (b). The spatially asymmetric enhancement of the carrier concentrations is due to the difference in the effective mobility constants $\mu_{n}$ and $\mu_{p}$. Similar to the grain boundary potential reduction, the excess charges lead to a reduction in the voltage drop of a $p n$-junction. While the Fermi levels are pinned at the ohmic contacts, a reduction of the $p n$-junction voltage will be observed in a limited field of view. As an example, the inset in Fig. 7(d) shows an enlarged view of the vicinity of the junction comparable to the field of view of a few $\mu \mathrm{m}$ in holographic TEM imaging at medium magnification. Figure 8 displays the voltage drop in a field of view of $2 \mu \mathrm{m}$ around the junction in dependence on the generation rate for dopant densities $N_{0}$ between $1 \times 10^{15} \mathrm{~cm}^{-3}$ and $1 \times 10^{17} \mathrm{~cm}^{-3}$.

A significant reduction in the junction voltage is recorded for generation rates $G$ in a TEM between $1 \times 10^{23} \mathrm{~cm}^{-3} \mathrm{~s}^{-1}$ and $1 \times 10^{24} \mathrm{~cm}^{-3} \mathrm{~s}^{-1}$ only for dopant densities well below $1 \times 10^{17} \mathrm{~cm}^{-3}$.

\section{DISCUSSION}

The numerical simulation presented in the previous section demonstrates the effect of the electron-generated excess charge carriers on the equilibrium electrostatic potential of defects and potential gradients in silicon. Whereas a grain boundary potential barrier suffers from a strong decrease in the barrier height, the voltage drop around the $p n$-junction is little affected for meaningful doping levels that are relevant for application. The distortion in the potential maps is related to the extrinsic excess charge carrier population in combination with their spatial separation and trapping at defect states. It has thus to be distinguished from precision limitations in spatial and phase resolution in the instrumental acquisition procedure and the quantitative holographic phase reconstruction, which were discussed elsewhere. ${ }^{4,5}$ The following discussion will be restricted to the extrinsic effects in holographic imaging experiments for indirect band-gap materials and focuses on general observations on the role of the sur-

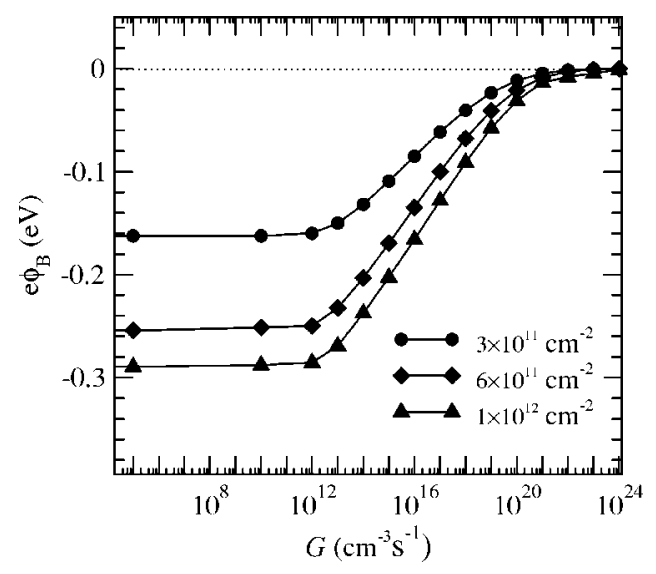

FIG. 6. Decrease of the grain boundary potential energy barrier height $e \phi_{\mathrm{B}}$ upon increase of the excess charge carrier generation rate $G$ plotted for various interfacial trap state densities $D$ at the grain boundary (solid symbols). 

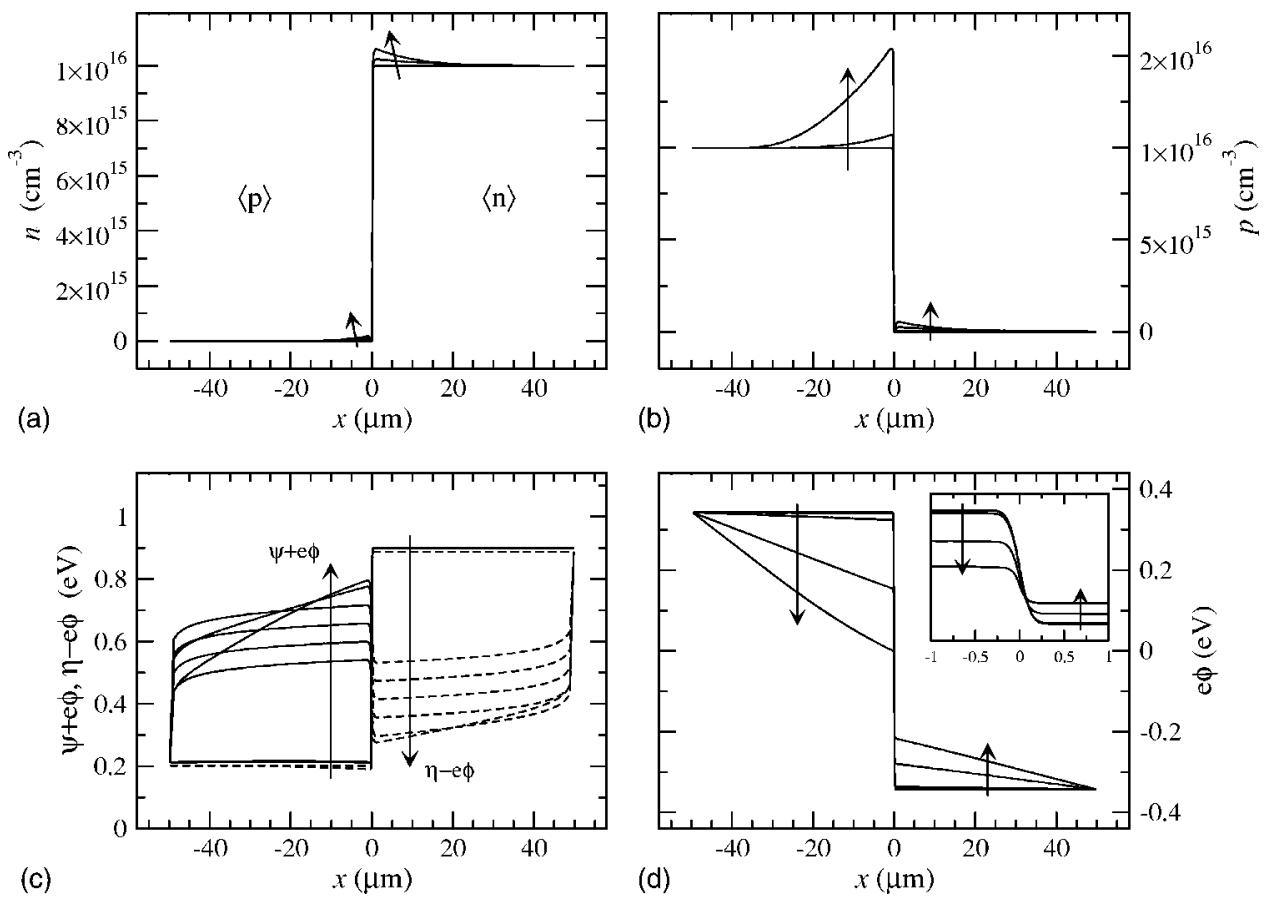

FIG. 7. The effect of extrinsic charge carrier generation on (a) the density of free electrons $n$, (b) the density of free holes $p$, (c) the electron electro-chemical potential $\eta-e \phi$ (dashed lines) and the hole electro-chemical potential $\psi+e \phi$ (solid lines) and (d) the potential energy $e \phi$ at a $p n$-junction with a dopant density $N_{0}=1 \times 10^{16} \mathrm{~cm}^{-3}$. The arrows indicate the trend upon increasing generation rate $G$. Curves are plotted for $1 \times 10^{10} \mathrm{~cm}^{-3} \mathrm{~s}^{-1}, 1 \times 10^{20} \mathrm{~cm}^{-3} \mathrm{~s}^{-1}, 1 \times 10^{21} \mathrm{~cm}^{-3} \mathrm{~s}^{-1}, 1 \times 10^{22} \mathrm{~cm}^{-3} \mathrm{~s}^{-1}, 1 \times 10^{23} \mathrm{~cm}^{-3} \mathrm{~s}^{-1}, 1 \times 10^{24} \mathrm{~cm}^{-3} \mathrm{~s}^{-1}$, and $2 \times 10^{24} \mathrm{~cm}^{-3} \mathrm{~s}^{-1}$. The inset in (d) shows an enlarged part with a width of $2 \mu \mathrm{m}$ around the junction.

faces of the TEM samples and the observed potential map distortions in device structures.

One should keep in mind that the basic transport equations underlying the numerical simulations are models imitating the real process in a qualitative and more or less accurately in a quantitative sense. Here, the model was applied to silicon, a semiconductor material with indirect band gap and defect mediated recombination. Results are, therefore, representative only for a similar kind of material, and they are not directly transferable, e.g., to direct band-gap semiconductors or insulators.

The influence of a defect-rich surface structure on quantitative potential maps is fully recognized in the experimental procedures, ${ }^{1-3,6}$ without, however, giving a clear picture. Rau et al. calibrated surface depletion layers ("dead layers") by measuring the thickness dependence of the electron wave phase shift. ${ }^{2}$ McCartney et al. and Gribelyuk et al. did not find dead layers, instead coating of the TEM samples with a few $10 \mathrm{~nm}$ thick carbon layers in conventionally $\mathrm{Ar}$ ion milled samples is reported to prevent from surface charging. 1,6

The special case of idealized amorphous silicon surface layers in Sec. III A shows that ion beam damaged surfaces can, under certain circumstances, be considered as passive. Surface charging is closely linked to the charging of interface trap states at a grain boundary. Therefore, the surface Fermi levels are affected by the excess charge carrier density in the same manner as the grain boundary interface Fermi levels. At high excess charge carrier concentration, it is unlikely that the Fermi levels will be pinned by the surface trap states. Similar to the interface states at the grain boundary, the sur- face trap states will charge with the tendency to maximize the recombination currents. The detailed charge state of the trap states at the surface depends on their energetic position, their capture cross-sections for electrons and holes and the dopant density and there will hardly be a general rule for the experiment.

For the purpose of device characterization, one should distinguish between built-in potential gradients due to doping inhomogeneities and electrically active defects. Built-in potential gradients separate minority and majority charge carriers and increase the recombination currents at the contacts.

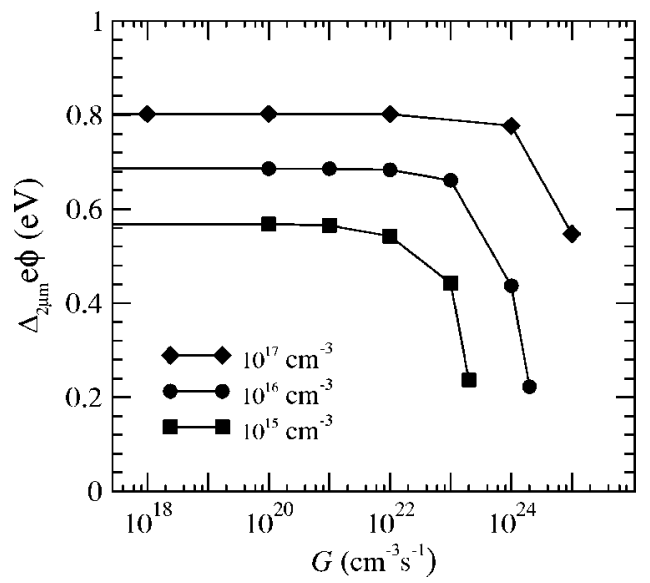

FIG. 8. Decrease of the $p n$-junction potential energy $\Delta_{2 \mu \mathrm{m}} e \phi$ over a distance of $2 \mu \mathrm{m}$ around the junction in dependence on the pair generation rate $G$. The data is given for base dopant densities $N_{0}$ between $1 \times 10^{15} \mathrm{~cm}^{-3}$ and $1 \times 10^{17} \mathrm{~cm}^{-3}$. 
This leads to an effective reduction of the excess charge carrier density in the illuminated region of interest. In contrast, isolated defects do not guide excess charge carrier to the contacts but attract minority carriers. The resulting change in the charge state and the quasi-Fermi level shift maximizes the recombination current at the trap states for the given conditions. Therefore, a holographic phase map translated into a potential map around defects is more sensitive to excess charge carrier generation in the electron beam than those around built-in potential gradients. The barrier height for the grain boundary defects in silicon decreases even below the instrumental sensitivity limit for off-axis holography of approximately $0.1 \mathrm{eV}^{4}$ at a rather low electron-hole pair generation rate higher than $G=1 \times 10^{20} \mathrm{~cm}^{-3} \mathrm{~s}^{-1}$. A similar breakdown of the potential barrier of a grain boundary was calculated analytically for the case of optical light excitation in poly-crystalline silicon. ${ }^{19}$ Even low dose illumination in the TEM and modified material parameters like smaller charge carrier lifetime $\tau$, e.g., in direct band-gap semiconductors with radiative recombination or in radiation damaged samples, are unlikely to change this fundamental result.

In the same manner, distinct contrast variations in holographic phase images of dislocations or heterogeneous interfaces associated with trap states have to be assessed carefully. In addition to a structural and compositional inhomogeneity, the electro-chemical potential of the charge carriers will adjust to maximize the recombination currents for excess charge carriers, similar to the case of the grain boundary demonstrated in Sec. III B. It must be stressed at this point, that there is no contradiction between the distorted electrostatic potential and a strong signal of dislocations in electron beam induced current (EBIC) experiments, precisely because the EBIC technique detects the enhanced recombination currents at defects by the loss in the carrier collection at sample contacts.
The impact of electron-hole pair generation is less critical for pure dopant gradients like in the case of the $p n$-junctions. Due to the smaller excess charge carrier concentrations at the same electron-hole pair generation rates $G$ compared to the case of defects in a homogeneous material, distortions of the potential map are expected only for the lower dopant densities for which experimental data are not available so far. However, the potential maps around heterogeneous interfaces like metallization contacts with electrically active defects cannot be interpreted directly on the basis of dopant densities.

\section{CONCLUSIONS}

Excess charge carrier generation in the electron beam alters the electrostatic potential in TEM samples. Most significant are changes in the electrostatic potential around defects associated with trap states. The potential barrier height of a grain boundary in silicon is reduced below the sensitivity limit of off-axis holographic phase imaging in the real experiment. Potential maps of dopant gradients in otherwise homogeneous material are affected only at lower dopant concentrations smaller than $10^{17} \mathrm{~cm}^{-3}$.

\section{ACKNOWLEDGMENTS}

We thank M. Lehmann, IAPD Technical University of Dresden, for giving us the possibility to do the off-axis holography experiments and for discussion. We thank J.H. Werner, IPE University of Stuttgart, for providing the silicon bicrystal samples. We also thank P. Schwander and A. Ourmazd, IHP Microelectronics Frankfurt (Oder) for the support in the prearrangement of studying grain boundary defects by holography.
${ }^{1}$ M. R. McCartney, M. A. Gribelyuk, Jing Li, P. Ronsheim, J. S. McMurray, and D. J. Smith, Appl. Phys. Lett. 80, 3213 (2002).

${ }^{2}$ W. D. Rau, P. Schwander, F. H. Baumann, W. Höppner, and A. Ourmazd, Phys. Rev. Lett. 82, 2614 (1999).

${ }^{3}$ A. C. Twitchett, D. E. Dunin-Borkowski, and P. A. Midgley, Phys. Rev. Lett. 88, 238302 (2002).

${ }^{4}$ W. J. de Ruijter and J. K. Weiss, Ultramicroscopy 50, 269 (1993).

${ }^{5}$ A. Harscher and H. Lichte, Ultramicroscopy 64, 57 (1996).

${ }^{6}$ M. A. Gribelyuk, M. R. McCartney, Jing Li, C. S. Murthy, P. Ronsheim, B. Doris, J. S. McMurray, S. Hegde, and D. J. Smith, Phys. Rev. Lett. 89, 025502 (2002).

${ }^{7}$ M. Gajdardziska-Josifovska, M. R. McCartney, W. J. de Ruijter, D. J. Smith, J. K. Weiss, and J. M. Zuo, Ultramicroscopy 50, 285 (1993).

${ }^{8}$ S. Selberherr, Analysis and Simulation of Semiconductor Devices (Springer-Verlag, Wien, 1984).

${ }^{9}$ The Physics of Hydrogenated Amorphous Silicon, edited by J. D. Joannopoulos and G. Lucovsky (Springer, Berlin, 1984).

${ }^{10}$ R. M. Barnett, C. D. Carone, D. E. Groom, T. G. Trippe, C. G. Wohl, B. Armstrong, P. S. Gee, G. S. Wagman, F. James, M. Mangano et al., Phys. Rev. D 54, 1 (1996).
${ }^{11}$ W. Schockley and W. T. Read, Phys. Rev. 87, 835 (1952).

${ }^{12}$ R. N. Hall, Phys. Rev. 87, 387 (1952).

${ }^{13}$ S. M. Sze, Physics of Semiconductor Devices (Wiley, New York, 1981).

${ }^{14}$ C. M. Snowden, Introduction to Semiconductor Device Modelling (World Scientific, Singapore, 1987).

${ }^{15}$ The carrier mobility depends on the doping level. The mobility is roughly one order of magnitude smaller than given in the table for $N_{A}=10^{19} \mathrm{~cm}^{-3}$. However, the conclusions drawn from Sec. III A are not affected since the diffusion length at $N_{A}$ $=10^{19} \mathrm{~cm}^{-3}$ still remains much larger than the sample thickness and therefore the peak excess charge carrier concentrations are essentially unchanged.

${ }^{16}$ D. J. Barber, Ultramicroscopy 52, 101 (1993).

${ }^{17}$ C. G. Van de Walle and L. H. Yang, J. Vac. Sci. Technol. B 13, 1635 (1995).

${ }^{18}$ C. R. M. Grovenor, Microelectronic Materials (Institute of Physics, London, 1989).

${ }^{19}$ C. C. Card and E. S. Yang, IEEE Trans. Electron Devices 24, 397 (1977). 